\title{
Susac syndrome as a multidisciplinary diagnostic challenge
}

\author{
Klaudia Sapko ${ }^{1, A-D \oplus}$, Anna Jamroz-Wiśniewska ${ }^{1, A-C, E-F \oplus}$, Anna Woźniak ${ }^{2, B-C, E \oplus,}$ \\ Michał Marciniec ${ }^{1, A, C, E \oplus}$, Marcin Kulczyński ${ }^{1, A-B, E \oplus}$, Marzena Janczarek ${ }^{3, B-C \oplus}$, Robert Rejdak ${ }^{2, E-F} \oplus$, \\ Konrad Rejdak ${ }^{1, E-F} \oplus$ \\ ${ }^{1}$ Chair and Department of Neurology, Medical University, Lublin, Poland \\ ${ }^{2}$ Department of General Ophthalmology, Medical University, Lublin, Poland \\ ${ }^{3}$ Department of Interventional Radiology and Neuroradiology, Medical University, Lublin, Poland \\ A - Research concept and design, B - Collection and/or assembly of data, C - Data analysis and interpretation, \\ $D$ - Writing the article, E - Critical revision of the article, F - Final approval of article
}

Sapko K, Jamroz-Wiśniewska A, Woźniak A, Marciniec M, Kulczyński M, Janczarek M, Rejdak R, Rejdak K. Susac syndrome as a multidisciplinary diagnostic challenge. Ann Agric Environ Med. 2022; 29(1): 157-161. doi: 10.26444/aaem/136524

\begin{abstract}
Introduction. Susac syndrome (SuS) is a disease manifested as the clinical triad of encephalopathy, branch retinal artery occlusion, and loss of sensory neural hearing.

Case Report. The case is presented of a 28 -year-old patient hospitalized due to visual impairment of the left eye, and whose hearing and neuropsychiatric disorders had appeared two years earlier. Magnetic resonance imaging demonstrated lesions located in the white matter and along the corpus callosum. An audiogram showed bilateral sensory neural hearing loss. Fluorescein angiography examination revealed branch retinal artery occlusion of the left eye. Based on the clinical picture and results of tests, the diagnosis of SuS was made. Despite the use of steroid and immunosuppression therapy the disease progressed.

Conclusions. The prognosis for SuS depends on the early diagnosis and implementation of treatment. It should be underlined that in case of hearing loss or encephalopathy of unknown cause, SuS should always be excluded.
\end{abstract}

\section{Key words}

Susac syndrome, branch retinal artery occlusion, encephalopathy, sensory neural hearing loss

\section{INTRODUCTION}

Susac syndrome $(\mathrm{SuS})$ is a rare autoimmune disorder first described by John O. Susac et al. in the USA in 1979 [1]. It is characterized by a clinical triad of encephalopathy, visual disturbance resulting from branch retinal artery occlusion (BRAO), and sensory neural hearing loss (SNHL) [2]. The disease occurs mostly in females aged 20-40 years of age. The etiology of this disease is unknown, but most likely CD8 + T-cell-mediated endotheliopathy, leading to micro-infarcts of the precapillary arterioles of the brain, inner ear and retin, is a key disease mechanism in SuS [3]. Diagnosis is based on clinical symptoms and the results of additional tests, such as magnetic resonance imaging (MRI), audiometry and retinal fluorescein angiography (FA) [4]. However, the disease may be monocyclic, may follow a polycyclic or chronic-progressive course, and the symptoms may appear simultaneously or, more often, successively, which makes diagnosis difficult [5]. A case of SuS is reported which for a long time was undiagnosed, until a complete clinical triad of this disease was performed.

\section{CASE REPORT}

A 28-year-old woman was admitted to the Neurology Department due to severe visual disturbances in the

Address for correspondence: Klaudia Sapko, Chair and Department of Neurology, Medical University, Lublin, Poland

E-mail: klaudia.sapko@gmail.com

Received: 20.03.2021; accepted: 10.05.2021; first published: 26.05.2021 left eye which had lasted for seven days. The patient had been admitted three days earlier to the Department of General Ophthalmology, where nystagmus and visual field defects were found in both eyes. The defects were more pronounced in the left eye as a result of superior temporal branch retinal artery occlusion (BRAO) (Fig. $1 \mathrm{~A}, \mathrm{~B})$. In the fundus examination, obstruction of the branch of the central retinal artery, ischemic and oedematous retina in the superior temporal quadrant and abnormal diameter of supratemporal branch retinal vein (thinner near the disc and thicker at the periphery) of the left eye, and the nearly normal appearance of the right eye were revealed (Fig. $1 \mathrm{C}$, D). FA confirmed BRAO in the left eye and showed focal nonperfused peripheral retinal arterioles in the right eye (Fig. 2 A, B). However, despite these ischemic changes, the best corrected visual acuity (BCVA) in both eyes was normal: $\mathrm{BCVA}=1.0$. In addition, due to progressive bilateral hearing impairment for 1.5 years, more severe on the right side, the patient was equipped with a hearing aid in the right ear; however, it should be noted that the patient, despite her hearing loss of unknown cause which had occurred at a young age, was not further diagnosed. Additionally, less than two years earlier the patient had been hospitalized in a Neuropsychiatric Hospital due to a psychotic episode, since which the patient has been under the control of a Psychiatric Clinic and was being treated with antipsychotics medication. Neurological examination at admission did not reveal any other abnormalities except for the visual field defect of the left eye, nystagmus and bilateral hearing loss.

MRI at 1.5 Tesla demonstrated numerous lesions located periventricular, less subcortical and along the 


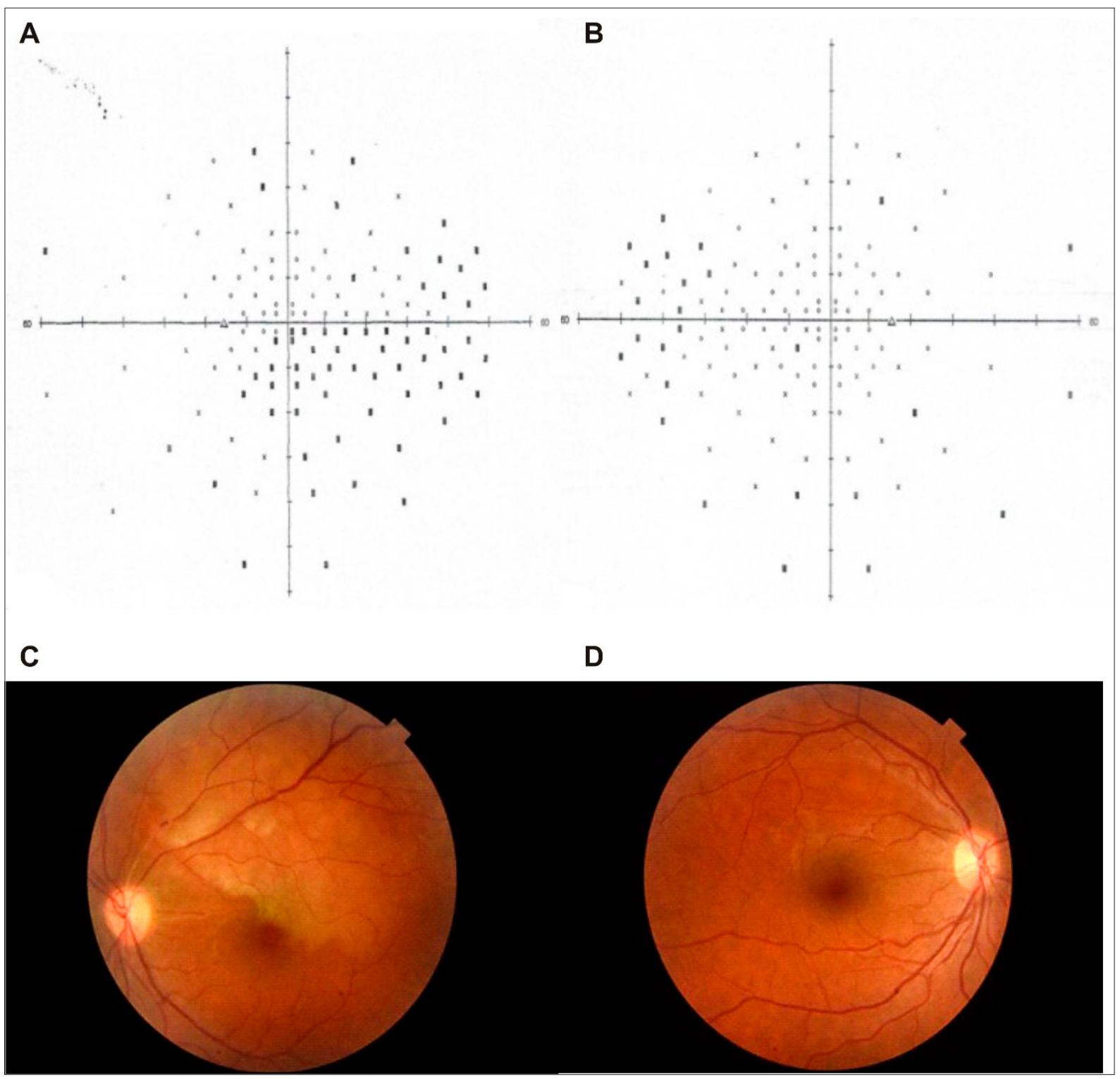

Figure 1. A) Visual field - left eye. B) Visual field - right eye. C) Fundus examination of the left eye - superior temporal branch retinal artery occlusion and ischemic and oedematous retina extending from the upper temporal vascular arcade nearby to the fovea (with foveal sparing). D) Fundus examination of the right eye

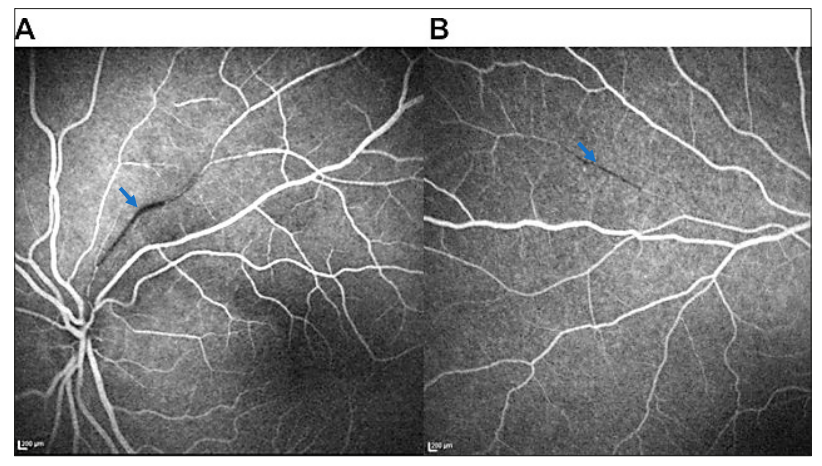

Figure 2. A) FA of the left eye - the focal hypofluoerscence of superior temporal branch retinal artery (blue arrow), which corresponds to reduced blood flow and retinal ischemia in the area supplied by the occluded artery. B) FA of the right eye - peripheral focal nonperfused retinal arterioles (blue arrow)

corpus callosum, in the form of continuous zones, which were high signal intensity on T2-weighted images (Fig. $3 \mathrm{~A}, \mathrm{~B})$. In the standard EEG study, disorganized basic function was found with slow theta and acute alpha waves located in the parieto-occipital-temporal region on both sides. Cerebrospinal fluid examination showed a mildly increased number of cells ( 15 cells/ $\mu \mathrm{l}$, normal range $0-5$ cells $/ \mu \mathrm{l})$, a slightly elevated protein level $(56 \mathrm{mg} / \mathrm{dl}$, normal range $15-45 \mathrm{mg} / \mathrm{dl}$ ) and absence of oligoclonal bands. An audiogram showed a bilateral SNHL, advanced for the right ear and moderate for the left ear (Fig. 4). In the psychological assessment of the patient, a mild dementia was found. A full systemic work-up excluded inflammation and infections (borreliosis, cytomegaly, toxoplasmosis), thrombophilia and connective tissue diseases. Aquaporin-4 antibodies were absent in the serum. Carotid and transcranial Doppler ultrasonography was performed but did not reveal any abnormalities.

Based on the clinical picture and tests results, with the confirmation of cerebral, cochlear and retinal involvement, the diagnosis of SuS was made. The patient was treated with intravenous methylprednisolone $1,000 \mathrm{mg}$ a day for seven days, followed by oral methylprednisolone $16 \mathrm{mg}$ per day and 


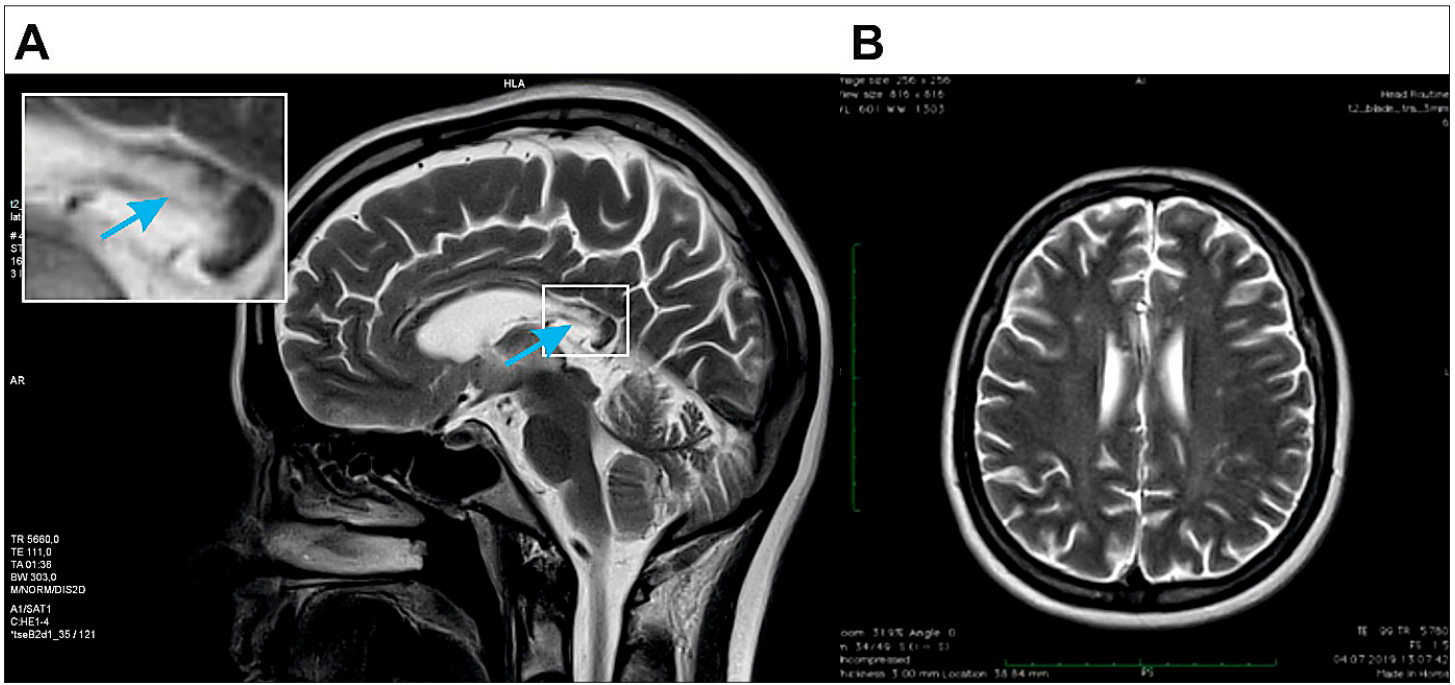

Figure 3. A) MRI-T2-weighted images with high signal intensity lesions in the form of continuous zones along the centrum callosum (sagittal projection). B) MRI - numerous high signal intensity lesions located periventricular, less subcortical in T2-weighted images (axial projection)

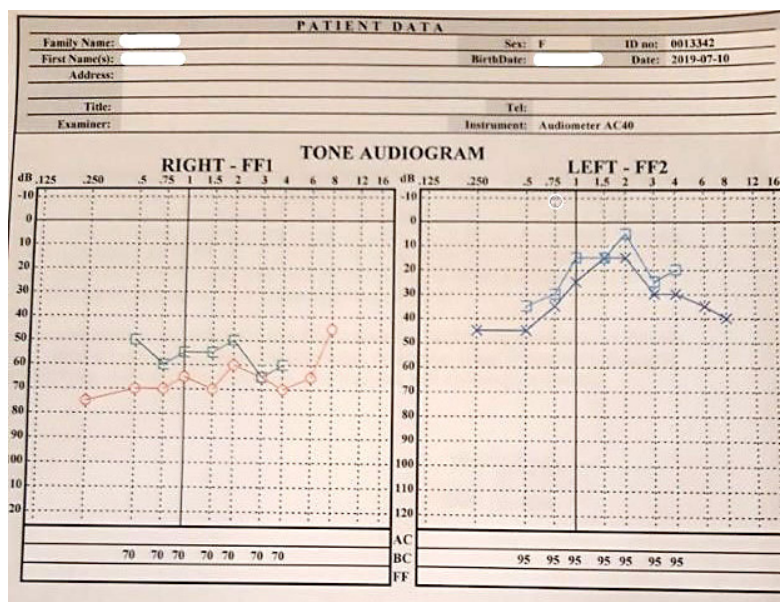

Figure 4. Audiogram - bilateral SNHL, advanced for the right ear and moderate for the left ear

acetylsalicylic acid $75 \mathrm{mg}$ per day. Unfortunately, there was no response to treatment and no improvement in the patient's condition. The patient was discharged home in a neurological state the same as on admission, with instructions to continue therapy with oral methylprednisolone and acetylsalicylic acid. In addition, due to the psychotic disorders, she was given quetiapine and fluoxetine. At a follow-up visit to the Neurological Outpatient Clinic, azathioprine was included in therapy at a dose of $150 \mathrm{mg}$ per day. A fundus follow-up examination one month after hospitalization showed retinal thinning of the left eye in the area provided by the affected upper temporal artery.

Seven months after the SuS diagnosis, the patient was re-hospitalized at the Neurologic Department due to the occurrence of right eye visual disturbances. A few days before hospitalization, the patient was examined ophthalmologically and an arterial occlusions located near the optic disc were found in the right eye (Fig. 5). In the left eye, irreversible retinal thinning in the upper temporal quadrant was noted (Fig. 6). During the interview, the patient admitted that she had not taken the prescribed medication (azathioprine) regularly. Neurological examination revealed incomplete auto- and allo-psychic orientation, memory

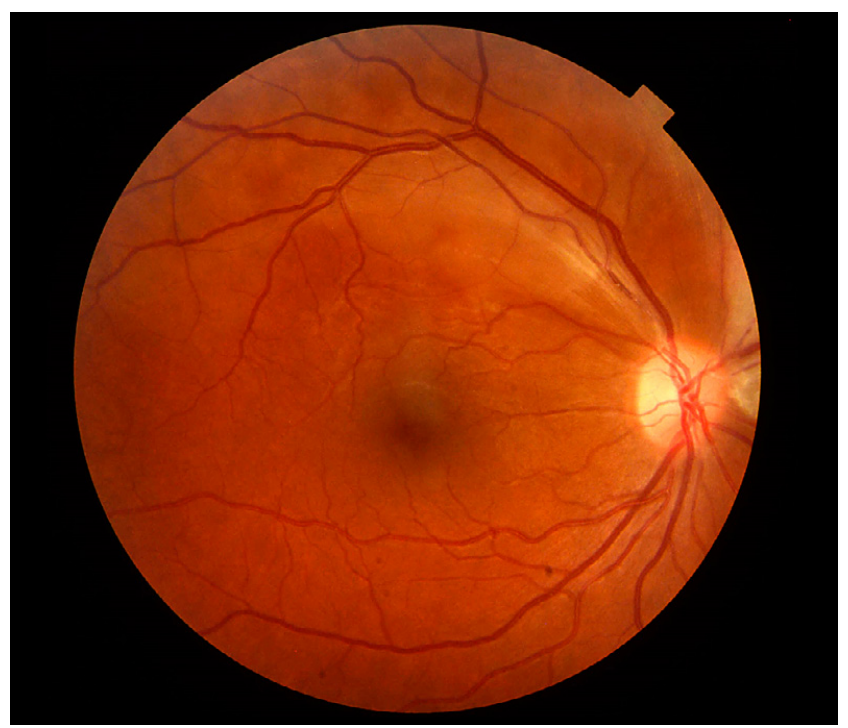

Figure 5. Acute manifestations of arterial occlusions in the right eye. Visual acuity was unaffected

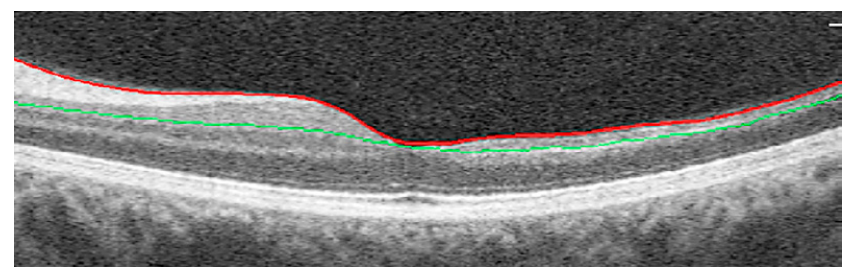

Figure 6. Optical coherent tomography (OCT) showing retinal thinning in the upper temporal quadrant of the left eye, six months after initial presentation

impairment, defect in the lower part of the visual field of the right eye, visual field defect as previously, and bilateral hearing loss, without other deviations. Intravenous methylprednisolone treatment with $1,000 \mathrm{mg}$ for four days, followed by oral methylprednisolone, was re-used. However, again there was no improvement after treatment. The patient was discharged home with a recommendation to continue therapy with oral methylprednisolone and azathioprine. The patient's condition observed during control visits has been stable but without improvement. 


\section{DISCUSSION}

SuS is a relatively rare vasculopathy first reported in 1973. Initially, it was described as small infarctions of cochlear, retinal, and encephalic tissues (SICRET) syndrome or retinopathy, encephalopathy, and deafness microangiopathy (RED-M) syndrome [6]. Eventually, the disease was described by Susac et al. in 1979 and named Susac's syndrome by William F. Hoyt in $1986[1,7]$.

The etiology of this disease has not yet been fully explained, but is most likely immune CD8 + T-cell-mediated endotheliopathy that leads to small vessel vasculitis, causing micro-infraction in the brain, retina and apical turn of the cochlea [3]. Autoimmune diseases are more prevalent in women, and similarly SuS was found to be more common in women than in men in a 3:1 ratio [8].

$\mathrm{SuS}$ is characterized by the triad of symptoms: encephalopathy, visual impairment and hearing loss; however, clinical triad is not universally present in SuS patients. The encephalopathy is manifested by headache, pyramidal and cerebellar syndrome, sensory deficiencies, dysarthria, aphasia, epileptic seizures, urinary insufficiency, cognitive impairment, personality and memory disorders, confusion, aggression, apathy, and depression [9]. Hearing loss, sometimes accompanied by vertigo and tinnitus, is usually bilateral and irreversible and is an indication for cochlear implantation [10]. BRAO can be one or two-sided, limited, or extensive and can cause black spots, photopsia or scintillating scotomas [4]. The disease may have monocyclic, polycyclic, or chronic-progressive course. The classic triad of $\mathrm{SuS}$ is absent in $97 \%$ of patients at the beginning of the disease, and it may take months to two years between the onset of the first symptom and the full manifestation of the disease, which makes an early diagnosis difficult to establish, as in the case of the presented patient [5].

To diagnose SuS, a clinical picture and the results of additional tests such as brain MRI, FA and tonal audiometry are necessary [4]. MRI is the basic neuroimaging examination of choice for SuS diagnosing and it demonstrates numerous, multifocal, and different sized high signal intensity ischemic lesions on T2-weighted and FLAIR images in the white and grey matter. These lesions are primarily located in the central part of the corpus callosum, as well as the subcortical, periventricular, thalamus, basal ganglia, cerebellum, and brainstem. Changes in the corpus callosum are described as micro-infarct lesions with specific images known as snowballs. They are considered pathognomonic for SuS, and when observed, there should be a high suspicion of SuS, especially in the presence of auditory and ophthalmological disorders. It is worth remembering that the lesions visible in MRI in SuS may imitate those present in multiple sclerosis (MS) or acute disseminated encephalomyelitis (ADEM). However, involvement of the grey matter and the presence of lesions in the fibres of the central part of the corpus callosum with relative sparing of the peripheral fibres, allows differentiating SuS from MS and ADEM [11]. In FA, BRAO is observed, which in the acute form of the disease may occur with pseudoemboli known as Gass plaques, present in the central segments of the retinal arterioles, accompanied by characteristic vascular staining and fluorescein leakage at sites remote from the actual BRAO. The changes are usually bilateral, may appear early or at a later stage of the disease, and in many cases enable the final diagnosis to be made [12].
In the described case, in FA there was observed only one small, typical focal hyperfluorescence of the arterial vessel wall in the left eye, with more pronounced branch retinal artery occlusions with non-filling of retinal vessels. However, FA was not performed at the time of reccurence.

Optical coherence tomography is an additional test that reveals damage to the retinal nerve fibre layer which is greater than that observed in MS [13]. In the presented case, retinal thinning in the left eye was caused by an acute ischemia due to BRAO. The tonal audiometry test is used to confirm the presence of SNHL in which hearing impairment involving the low and mid-frequency tones is demonstrated [14]. Cerebrospinal fluid assessment is a non-specific examination in the diagnosis of SuS; however, increase in protein concentration and slight lymphocytic pleocytosis is observed in most cases. Oligoclonal bands are absent, which allows differentiation from MS [15]. In addition to MS and ADEM, there are several differential diagnoses, including systemic lupus erythematosus, vasculitis, thromboembolic stroke, Ménière disease, complicated migraine, antiphospholipid syndrome, sarcoidosis, neuroBehçet disease, Marchiafava-Bignami disease and cerebral autosomal-dominant arteriopathy with subcortical infarcts and leukoencephalopathy [16].

There are currently no uniform guidelines for treatment in SuS. Due to the likely autoimmune etiology of the disease, it is important to introduce immunomodulatory treatment, which, if used at an early stage of the disease, can significantly improve the prognosis. Aggressive intravenous glucocorticosteroid therapy is recommended as first-line treatment in the acute phase of the disease, followed by oral steroid therapy at a lower dose, depending on the patient's condition and response to treatment. Steroids in high doses are used as a standard treatment in the exacerbation of many neurological diseases for a period of a few days (sometimes with subsequent taper). Usually it does not cause severe side-effects. No increased mood disorders or biochemical abnormalities were observed in the presented patient. Together with steroids, supplementation of potassium, calcium and vitamin $\mathrm{D}$ was applied as a preventive therapy. Immunoglobulins or plasmapheresis may also be considered if steroid therapy is ineffective. In long-term treatment, immunosuppressive drugs, such as azathioprine, mycophenolate mofetil, cyclophosphamide, cyclosporine, methotrexate and rituxymab, are mainly used. Acetylsalicylic acid may also be used to reduce the procoagulopathic state. In the current case, intravenous immunoglobulins could have been used to induce a remission; however, they were not applied becauses methylprednisolone with azathioprine seemed to be sufficient.

The last relapse of the disease was associated with a temporary break (due to the patient's poor compliance in the beginning) in immunosuppressive therapy with azathioprine. It is necessary to educate patients about the side-effects of using immunosuppressive drugs, the risk of infertility, and the need for preventive vaccinations. In patients with severe hearing loss, cochlear implantation is indicated [17].

The prognosis for SuS depends on the early diagnosis and implementation of treatment. Unfortunately, the occurrence of symptoms at intervals, the lack of clear diagnostic criteria, misdiagnosis with other diseases, mainly MS, and the multidisciplinary nature of the disease makes the diagnosis of SuS very difficult and delays the inclusion of proper 
therapy which, in turn, leads to permanent damage to the nervous system, which is what happened in the patient in the presented case report.

\section{CONCLUSIONS}

SuS should be suspected in every patient, especially at a young age, who develops progressive encephalopathy and neuropsychiatric symptoms associated with hearing and vision disorders. It should be remembered that SuS is a multidisciplinary problem, often undiagnosed or misdiagnosed as it mimics many other diseases, especially MS. Moreover, the typical triad of symptoms usually does not appear simultaneously, which delays the diagnosis and implementation of aggressive treatment, which when used at an early stage significantly improves the prognosis.

\section{REFERENCES}

1. Susac JO, Hardman JM, Selhorst JB. Microangiopathy of the brain and retina. Neurology. 1979; 29(3): 313-316. https://doi.org/10.1212/ wnl.29.3.313

2. Nazari F, Azimi A, Abdi S. What is Susac syndrome? - A brief review of articles. Iran J Neurol. 2014; 13(4): 209-214.

3. Gross CC, Meyer C, Bhatia U, et al. CD8+ T cell-mediated endotheliopathy is a targetable mechanism of neuro-inflammation in Susac syndrome. Nat Commun. 2019; 18; 10(1): 5779. https://doi.org/10.1038/ s41467-019-13593-5

4. Pereira S, Vieira B, Maio T, et al. Susac's Syndrome: An Updated Review. Neuroophthalmology. 2020; 44(6): 355-360. https://doi.org/10.1080/0 1658107.2020.1748062

5. Jarius S, Kleffner I, Dörr JM, et al. Clinical, paraclinical and serological findings in Susac syndrome: an international multicenter study.
J Neuroinflammation. 2014; 8; 11: 46. https://doi.org/10.1186/17422094-11-46

6. Murata Y, Inada K, Negi A. Susac syndrome. Am J Ophthalmol. 2000; 129(5): 682-684. https://doi.org/10.1016/s0002-9394(00)00377-9

7. Susac JO. Susac's syndrome: the triad of microangiopathy of the brain and retina with hearing loss in young women. Neurology. 1994; 44(4): 591-593. https://doi.org/10.1212/wnl.44.4.591

8. Dorr J, Krautwald S, Wildemann B, et al. Characteristics of Susac syndrome: a review of all reported cases. Nat Rev Neurol. 2013; 9(6): 307-316. https://doi.org/10.1038/nrneurol.2013.82

9. García-Carrasco M, Mendoza-Pinto C, Cervera R. Diagnosis and classification of Susac syndrome. Autoimmun Rev. 2014; 13(4-5): 347-350. https://doi.org/10.1016/j.autrev.2014.01.038

10. Hardy TA, Taylor RL, Qiu J, et al. The neuro-otology of Susac syndrome. J Neurol. 2020; 267(12): 3711-3722. https://doi.org/10.1007/s00415020-10086-0

11. Marrodan M, Correale J, Alessandro L, et al. Susac Syndrome: A differential diagnosis of white matter lesions. Mult Scler Relat Disord. 2017; 15: 42-46. https://doi.org/10.1016/j.msard.2017.04.007

12. Heng LZ, Bailey C, Lee R, et al. A review and update on the ophthalmic implications of Susac syndrome. Surv Ophthalmol. 2019; 64(4): 477485. https://doi.org/10.1016/j.survophthal.2019.01.007

13. Ringelstein M, Albrecht P, Kleffner I, et al. Retinal pathology in Susac syndrome detected by spectral-domain optical coherence tomography. Neurology. 2015; 85(7): 610-8. https://doi.org/10.1212/ WNL.0000000000001852

14. Patel VA, Dunklebarger M, Zacharia TT, et al. Otologic manifestations of Susac syndrome. Acta Otorhinolaryngol Ital. 2018; 38(6): 544-553. https://doi.org/10.14639/0392-100X-2166

15. Vodopivec I, Venna N, Rizzo JF, et al. Clinical features, diagnostic findings, and treatment of Susac syndrome: a case series. J Neurol Sci. 2015; 357(1-2): 50-57. https://doi.org/10.1016/j.jns.2015.06.063

16. Vishnevskia-dai V, Chapman J, Sheinfeld R, et al. Susac syndrome: clinical characteristics, clinical classification, and long-term prognosis. Medicine (Baltimore). 2016; 95(43): e5223. https://doi.org/10.1097/ MD.0000000000005223

17. Egan RA. Diagnostic Criteria and Treatment Algorithm for Susac Syndrome. J Neuroophthalmol. 2019; 39(1): 60-67. https://doi. org/10.1097/WNO.0000000000000677 